Itinéraires Itinéraires

Littérature, textes, cultures

2016-3 | 2017

"Banlieues » : entre imaginaires et expériences

\title{
Entretien avec Rachid Santaki
}

Interview with Rachid Santaki

\section{Serena Cello}

\section{(2) OpenEdition}

\section{Journals}

Édition électronique

URL : http://journals.openedition.org/itineraires/3544

DOI : 10.4000/itineraires.3544

ISSN : 2427-920X

Éditeur

Pléiade

\section{Référence électronique}

Serena Cello, « Entretien avec Rachid Santaki », Itinéraires [En ligne], 2016-3 | 2017, mis en ligne le 15 juillet 2017, consulté le 01 mai 2019. URL : http://journals.openedition.org/itineraires/3544 ; DOI : 10.4000/itineraires.3544

Ce document a été généré automatiquement le 1 mai 2019.

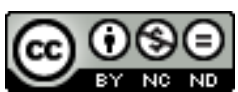

Itinéraires est mis à disposition selon les termes de la licence Creative Commons Attribution - Pas d'Utilisation Commerciale - Pas de Modification 4.0 International. 


\title{
Entretien avec Rachid Santaki
}

\author{
Interview with Rachid Santaki
}

\author{
Serena Cello
}

\section{NOTE DE L'ÉDITEUR}

Rachid Santaki a participé en tant qu'écrivain invité au colloque «Banlieues/Periferie : quelles représentations contemporaines des quartiers "sensibles" ? ». Il vit en banlieue parisienne, où ses activités d'écrivain et d'éducateur, mais aussi son engagement incessant dans l'organisation d'activités culturelles et sociales, le placent dans une position privilégiée pour fournir un regard avisé sur la réalité multiple et mouvante de la vie dans les zones urbaines périphériques. Il a publié des romans dont, notamment, Les anges s'habillent en caillera (Moisson rouge, 2011), Flic ou Caillera (Éd. du Masque, 2013), Business dans la cité (Seuil, 2014), La Légende du 9-3 (Ombres Noires, 2016), et un essai, La France de demain (Wildproject), avec Brahim Chikhi en 2015.

Entretien réalisé par Serena Cello à l'Alliance française de Gênes, le 16 septembre 2015. Enregistrement sonore transcrit par Elisa Bricco et Nancy Murzilli.

SERENA CELLO : À propos de la représentation des banlieues, pourquoi avez-vous choisi le polar en forçant encore plus le trait sur les côtés les plus violents et sombres ? Ne s'agit-il pas d'un risque de perpétuation des clichés sur la banlieue?

Rachid Santaki : Souvent on me pose la question comme si j'avais un rôle d'élu, c'est-àdire que si vous traitez de la banlieue comme sujet vous avez une responsabilité. Ce qui n'est pas le cas car nous sommes dans le cadre de la fiction. Je trouve intéressant de passer par la fiction parce qu'elle permet plus de liberté. En plus, la fiction permet de choisir la couleur. Lors de mes premiers entretiens avec les journalistes, on me demandait si je ne peignais pas une banlieue trop noire, donc négative. J'expliquais que, s'agissant de fiction, j'avais la possibilité de faire un choix sur la couleur de mes œuvres et je faisais souvent la comparaison avec le graffiti. Quand un artiste fait de la peinture, notamment du graffiti, il peut faire des lettrages colorés, du monochrome, etc. La 
fiction est aussi une possibilité qui donne la liberté de ne pas être assigné. Très souvent, quand on vient de banlieue, de périphérie, on vous fait croire que vous avez un rôle et que vous devez parler de manière positive de votre territoire. Comme je ne veux pas me limiter à cela, j'ai choisi la fiction.

s.c. : Vous mettez en scène une police assez immorale où le délinquant et le policier partagent les mêmes codes.

R.S. : Je voulais faire bouger les lignes. Le policier, le médecin, le délinquant sont avant tout des êtres humains avec leurs forces et leurs faiblesses. Donc ce n'est pas tout blanc ou tout noir, il y a des ambiguïtés, il y a des frontières qu'on dépasse ou qu'on ne dépasse pas. Quand on est en banlieue, on parle souvent de certains jeunes comme de délinquants, et je trouvais intéressant de déplacer le point de vue du côté de la police et de le démontrer.

Je vais vous citer un exemple : j'habite à Saint-Denis et le commissariat de Saint-Denis emploie 250 fonctionnaires de police. Il y a un an et demi j'ai eu une discussion avec un préfet de police à qui j'ai raconté qu'à Saint-Denis une équipe de la BAC ${ }^{1}$ "rackettait » les dealers. Pour résoudre la situation à leur manière, les jeunes du quartier se sont enfermés dans le parking, ils se sont battus à mains nues avec les policiers et les policiers ont été dominés. Le préfet ne voulait pas me croire, parce que pour lui un tel comportement de la part de policiers est inimaginable. Cette histoire a pourtant fini par ressortir, il y a moins de six mois, dans les médias : on a parlé de la BAC de Stains qui rackettait des dealers. Au commissariat de Saint-Denis les policiers sont débordés parce que là-bas il y a entre autres des problèmes de vol à la portière, il y a eu dernièrement un gros mouvement de toxicomanes : on a fermé les squats dans le $18^{\mathrm{e}}$ arrondissement de Paris et entre cinquante, et cent toxicomanes sont venus habiter dans des squats à Saint-Denis. Il y a quatorze quartiers à Saint-Denis, c'est une ville de 100000 habitants, avec des conflits de voisinage et énormément de problèmes. Donc, quand vous êtes policier et que vous êtes confronté à une telle pression il est difficile de rester « zen » et de ne pas franchir les limites, parce que vous êtes humain avant tout.

Lorsque je me documentais sur la police parce que je voulais donner corps à mes personnages, j'ai eu l'occasion de parler avec un commissaire qui m'a expliqué que depuis 1997, on a donné des objectifs aux policiers, on les a mis sous pression. À chaque changement de dirigeant politique, les objectifs changent et certains agents ont l'impression d'être ce qu'ils appellent les "égouts de la République »: on leur en demande beaucoup et certains finissent par craquer. Il y a un fort taux de suicide chez les policiers. Je ne voulais pas faire un policier à la Maigret avec sa pipe, cool, mais plutôt des policiers qui sont aussi sous pression, parce que la fonction publique, avec les réformes imposées, est confrontée aux difficultés qu'engendre la politique de résultat. Je pense que quand on est en banlieue on est confronté avec un monde qui est dur et on devient dur aussi. Il y a peut-être des policiers qui réussissent à être imperméables mais pour ça il faut avoir un bon équilibre familial. Ce qui m'intéresse avant tout c'est la façon dont l'humain gère ça.

s.c. : En lisant la trilogie des polars on voit une volonté d'interpeller le réel et de mettre en question le passé. Vous évoquez les événements du 17 octobre 1961 et les émeutes de 2005. Pourquoi avoir choisi de revenir sur ces deux épisodes?

R.S. : Quand j'ai écrit mon premier polar, Les Anges s'habillent en caillera ${ }^{2}$, je n'avais pas fait appel à un contexte "historique». Ensuite, j'ai rencontré d'autres auteurs, qui m'ont ouvert des voies vers cette réflexion. Dominique Manotti, par exemple, que 
j'apprécie énormément, qui est historienne et qui a été une militante. Elle m'a raconté son parcours de femme syndicaliste déçue qui s'est tournée vers l'écriture. En tant qu'historienne, elle utilise le polar en le situant dans un contexte historique. J'ai trouvé sa démarche intelligente et il m'a semblé important, à partir du moment où j'écris des romans qui se passent en banlieue, d'y insérer des faits historiques qui ont joué un rôle dans les événements que je raconte. Les faits du 17 octobre 1961 et les révoltes sociales de 2005 permettent de donner une profondeur à mes romans et de les ancrer dans la durée. Les éléments historiques forment le socle du roman.

s.c. : Pensez-vous que les événements de 2005 et surtout la fracture des événements du 17 octobre 1961 est encore présente dans la mémoire des Français ?

R.S. : Le conflit avec l'Algérie date d'il y a plus de 50 ans et il n'est toujours pas réglé. C'est très compliqué parce qu'il y a plusieurs conflits qui ne sont pas réglés. Le juge Trévidic ${ }^{3}$ l'explique bien dans son livre, où il raconte l'histoire d'un jeune qui est parti faire le jihad parce qu'il a vécu comme une injustice les récits de son oncle sur la guerre d'Algérie et le FLN. En France, on se trouve sur un territoire où des gens n'ont pas réglé leurs conflits, et c'est aussi ce qui crée un problème d'identité. En banlieue, aujourd'hui, les jeunes issus de l'immigration ont des difficultés à trouver leur place, ils ne savent pas vraiment pourquoi ils sont là. Nos parents ont des conflits et on grandit avec ça. Ça fait de nous des schizophrènes.

s.c. : Passons au style: vous utilisez un langage particulier, avec des phrases courtes et une cadence rapide se rapprochant du rap et du slam. Est-ce que vous pouvez nous parler de votre rapport avec ces genres musicaux et pourquoi vous les inscrivez dans votre écriture?

R.S. : Dans mon travail de construction du roman il y a différentes étapes. En premier lieu, je me concentre sur la structure. Je me demande ce que je vais raconter, quel est le propos et quel contexte historique je pourrais lui donner. Le héros des Anges s'habillent en caillera est un jeune qui n'a rien et qui veut tout mais qui ne sait pas pourquoi. Il court après l'argent, il fait de l'argent mais il ne sait plus pourquoi. Il n'a pas de projet et donc pas de socle. Il y a d'abord le thème et ensuite je le développe, je pose les personnages, ce qu'on appelle la bible des personnages, et à partir de là je fais un chapitrage.

J'écris ensuite les chapitres sur de la musique rap. En effet, j'écoute du rap tous les jours en allant au bureau et, inconsciemment, la sonorité, la musicalité avec laquelle je commence ma journée, rentre dans mon écriture. Cela se produit parfois dans les passages de narration, car je trouve que la sonorité donne du relief au récit. J'évite de faire entrer ce rythme dans les dialogues, en revanche, parce qu'il ne serait pas crédible que mes personnages se mettent à faire de la rime.

J'ai dirigé un magazine de rap pendant huit ans, j'écoute du rap depuis longtemps mais j'écoute aussi de la funk, de la soul, différents genres musicaux. Je pense que cela a un impact sur mon écriture. Au départ je disais que je faisais de la littérature hip-hop pour la sonorité et pour la façon dont j'en faisais la promo. L'aspect hip-hop de mes premiers romans est lié au collage d'affiches que je faisais la nuit. Lors de la parution de mes deux premiers romans, comme pour un parti politique ou un concert de rap, j'avais imprimé des affiches $80 \times 120 \mathrm{~cm}$, préparé de la colle avec de la fécule de pomme de terre et de la lessive de soude, je partais avec mon utilitaire et je collais dans la rue. Je faisais cela parce que je voulais me démarquer des autres auteurs. Plutôt que de 
travailler seulement sur l'écriture, j'allais aussi dans la rue afficher mes romans. Donc la culture hip-hop est dans la sonorité mais aussi sur le terrain.

s.c. : Dans vos polars, vous mettez en scène une jeunesse qui a perdu ses repères, dans une société où règne l'hyperconsommation, où tout est permis et où l'argent est la seule valeur. Pourriez-vous expliquer ce choix, votre rapport avec cette jeunesse et les associations pour les jeunes présentes dans les quartiers?

R.S. : Dans un récent entretien, j'expliquais que la République n'est plus dans les quartiers. C'est-à-dire que dans les quartiers il y a les gens qui travaillent, qui paient leurs impôts, etc., et puis il y a les autres personnes qui vivent dans une société parallèle, qui se débrouillent avec les stupéfiants, les trafics, les voitures. Pour un jeune, aujourd'hui, la "survie" c'est d'aller acheter une sacoche à 600 euros chez Louis Vuitton. Dans les quartiers, la « survie » c'est avoir un Louis Vuitton original et montrer qu'on a réussi en mimant les gens qui consomment du luxe, qui, eux, ont réellement les moyens. Du coup, cela demande de multiplier les revenus. Mes personnages se sont construit une culture parallèle, avec leurs codes (le rap, le sport, etc.) qui ne sont pas forcément ceux du grand public. Ces personnages ne correspondent pas par exemple aux représentations que donnaient des rappeurs comme Kaaris. Faute de motivation et de perspectives sociales, mes personnages, qui n'ont ni repères familiaux ni perspectives pour l'avenir, se focalisent sur l'argent: ils ont complètement assimilé notre société d'hyperconsommation et ils sont les premiers à vouloir y participer. Par conséquent, les jeunes sont dans l'hyper-violence, parce qu'ils refusent la condition sociale de leurs parents, ils refusent d'être au SMIC, même si cela doit les conduire en prison. J'interviens en maison d'arrêt, auprès de différents groupes, d'hommes, de femmes, de mineurs. En ce qui concerne les mineurs, cela fait quatre ans que j'interviens à Strasbourg, et je constate que je retrouve toujours les mêmes, qui récidivent. Ils sont pris dans un mécanisme qui les pousse à vouloir faire de l'argent à tout prix : faire un coup, gagner 30000 euros et aller les dépenser en quinze jours de vacances à Marrakech. Le problème des jeunes d'aujourd'hui n'est plus celui de leurs parents qui essayaient de nourrir une famille, mais de montrer qu'ils ont réussi en brillant aux yeux des autres.

Certains de mes personnages sont comme cela, mais il y en a aussi qui ont développé une culture, qui ont de l'humour, et qui ne ressemblent pas aux personnages qu'on voit dans les clips vidéo de rap.

Le problème de la famille est aussi toujours présent dans mes romans. La famille a souvent une structure monoparentale. Le père peut être là, ou effacé, ou absent. Aujourd'hui, très souvent aussi dans la réalité les enfants sont devenus les adultes, puisque les adultes ne prennent plus leurs responsabilités. Quand un "gamin » arrive à se procurer 30000 euros et à passer quinze jours à Marrakech, dans une famille où le père ne travaille plus, ou il a eu un accident de travail, où la mère fait des ménages, c'est l'enfant qui prend le dessus et qui devient le chef de famille. C'est aussi ce qui fait que les structures familiales ont changé.

Je vous raconte une anecdote que j'avais trouvée frappante: un jeune qui vend du «shit » m'a dit qu'une mère envoyait son fils de huit ans lui acheter sa dose. L'enfant qui vit cette situation, ne grandit pas dans une structure familiale normale. C'est un cas extrême, mais en général les repères, qui se situent désormais dans les modèles d'hyperconsommation hors de portée, ne correspondent plus à ceux des parents et par 
conséquent les parents sont destitués. À partir de là, il est intéressant aussi de voir comment les jeunes se construisent eux-mêmes leurs modèles et leurs références.

s.c. : Je propose de passer au dernier livre, La France de demain, publié en 2015, co-écrit avec Brahim Chikhi. Quelle est l'idée de départ de ce manifeste qui a comme sous-titre «Réconcilier République et banlieue »?

R.S. : En tant que romancier, comme certains chercheurs, mais sans leurs diplômes, je fais beaucoup de terrain. J'interviens en maisons d'arrêt, auprès de gens qui apprennent la langue française, pour des bailleurs qui me donnent des missions, par exemple, sur un parc de 500 logements où je dois, à travers l'organisation d'activités culturelles, prendre contact avec les éléments forts du quartier, créer une dynamique et aboutir à l'établissement d'un lien social. En fait, j'avais énormément d'expérience de terrain, mais je me suis rendu compte que, lorsqu'on est romancier, les gens ne prennent pas forcément au sérieux ce que l'on dit. Je me souviens d'un journaliste de Marianne qui avait lu un de mes romans et qui a dit que c'était du cliché, du TF1. En fait, il ne me connaissait pas, il ne savait pas que j'étais éducateur sportif, il ne connaissait pas mon vécu. Au début, j'ai utilisé la fiction pour ne pas me donner un rôle de représentant qui n'est pas le mien, mais cela ne me permettait pas de partager toutes ces expériences de terrain. C'est pourquoi le format de l'essai s'est imposé, car il pouvait me permettre d'aborder les thèmes que je rencontre dans la banlieue : l'école, le travail, la religion et le territoire.

À partir de ces quatre thèmes, j'ai tout simplement parlé de différentes expériences. Lorsque l'essai est sorti, il a eu beaucoup d'échos, auprès de gens qui ont constaté qu'il pouvait apporter des réponses simples à certaines problématiques, parce qu'il présentait des cas concrets de confrontation directe aux difficultés rencontrées dans la banlieue. Prenons par exemple le sujet de la religion qui est d'actualité en France. On s'inquiète aujourd'hui d'assister à une montée du communautarisme et ce problème est réel dans les quartiers. Pour moi l'explication de ce problème vient du fait que la République n'est plus dans les quartiers. Pour certains, l'équilibre ne vient plus que de la religion et donc les seuls interlocuteurs que vous pouvez avoir ce sont des imams. Alors il suffit que ces gens soient dans une dérive pour que l'impact sur les jeunes puisse être très dangereux. Lorsque je discute avec des jeunes qui ont le sentiment d'avoir une connaissance religieuse assez poussée, je leur explique que c'est très bien d'avoir ces connaissances, mais que de mon point de vue la religion est de l'ordre de l'intime. Alors pourquoi utiliser la religion pour résoudre des problèmes qui sont en fait des problèmes d'identité sociale? Dans les quartiers, la religion permet d'avoir une identité, une famille, elle compense le manque de véritable structure familiale. Dans l'essai, je montre que la religion est devenue une marque d'identité sociale, alors que cela relève en fait de l'intime. Lorsqu'on est confronté à l'autre, il faudrait pouvoir se rappeler qu'on est d'abord face à un humain qui possède ses propres convictions, mais aujourd'hui, à cause de cela il est très facile d'entrer en conflit. On se dirige en effet vers un communautarisme qui tend à créer des clivages sociaux. À partir du moment où on vit séparés en communautés étanches, on ne peut plus communiquer et composer ensemble, cela fige notre société.

Dans l'essai, je parle aussi de l'école. Je suis convaincu que les enfants c'est l'avenir et que si on n'a pas de diplôme ou tout au moins des enseignants à qui on donne des moyens, on ne pourra pas faire évoluer notre quartier. Il faut parvenir à transmettre aux jeunes la curiosité et la connaissance. Le rôle des enseignants n'est pas de faire leur 
éducation, mais parfois la rencontre avec un enseignant passionné par son travail peut jouer le rôle de déclic. Il peut apporter quelque chose à ces jeunes et les aider à sortir de l'emprise des images stéréotypées de la femme, de l'argent. Les jeunes qui n'ont pas la possibilité de faire des études vont se forger une culture qui passe beaucoup par le rap, le rap deviendra leur école.

L'essai avait pour objectif de faire partager ces expériences à travers un document qui ne soit pas un roman auquel on peut reprocher de ne pas représenter la réalité.

s.c. : Pourriez-vous nous parler de la question de l'habitat et du territoire que vous abordez dans votre essai?

R.S. : J'ai grandi à Saint-Ouen et j'ai fait ma scolarité à Saint-Denis où j'habite encore aujourd'hui, j'ai habité dix ans à La Courneuve. L'identité de la banlieue a disparu. La banlieue au départ ce sont de grandes barres, qui pour moi étaient des espèces de cages où on faisait des expériences avec des êtres humains. Je pense à Balzac, la barre des 4000. Des amis habitaient là-bas, et concrètement cela tombait en pièces, les parties communes se dégradaient très rapidement et pour les familles ce cadre de vie était véritablement catastrophique. Dans le cadre de la politique de la ville, de grands projets de rénovation urbaine ont commencé à être mis en place au milieu des années 1990 et se sont accélérés avec le travail de l'Agence nationale de rénovation urbaine. Cela a conduit à la disparition de ces singularités de la banlieue. Les grandes cités, comme les 4000, les 3000 et Saint-Rémi à Saint-Denis, ont disparu et un grand travail de rénovation a été fait. Dans le 77, dans les villes nouvelles, les nouveaux bâtiments se ressemblent tous. Il est vrai qu'il y a eu beaucoup de financements dédiés à l'aménagement du territoire et à l'amélioration de l'environnement, mais on n'a pas détruit les barres qui étaient dans la tête des habitants. Le ghetto est mental, il se construit avec tous les clichés liés à la banlieue. Les habitants sont toujours prisonniers de leur quartier parce qu'il leur manque une sorte d'éducation sociale, la curiosité et l'envie de participer à la vie sociale. Ils sont toujours en position de victimes mais ils ne sont pas à même de combattre ce sentiment et de sortir de leur condition d'exclus. Il faudrait qu'ils acquièrent une conscience politique et qu'ils restent dans les quartiers pour les faire progresser de l'intérieur.

Donc l'urbanisme a changé, mais à l'intérieur il n'y a pas d'évolution, et cette évolution ne peut pas venir de l'extérieur. C'est aux gens de se responsabiliser et de s'organiser mais c'est très difficile. En banlieue on vit au jour le jour parce qu'on a grandi sans se projeter ; le moyen et le long terme n'existent pas et il est donc difficile de construire et de changer les choses.

s.c. : Dans votre essai vous parlez de la responsabilisation des habitants des quartiers ? Mohamed Mechmache, par exemple, lorsqu'il a fondé le collectif ACLEFEU, a rencontré la difficulté d'engager les gens du quartier, même après les émeutes de 2005, dans un projet citoyen. Je me demande à travers quelles stratégies peut-on tenter de responsabiliser les habitants des quartiers en tant que citoyens?

R.S. : Je suis convaincu que désormais ni la politique ni le militantisme ne pourront engendrer une prise de conscience des habitants des banlieues. Ce qui peut faire évoluer les choses, c'est la fiction. Notamment, si on prend en considération l'impact du rap sur les jeunes et des représentations qu'il véhicule, je pense que la fiction peut permettre d'envisager la possibilité du changement. En effet, en mettant en scène des personnages issus de l'immigration dans des rôles valorisants comme ceux de présidents, d'avocats, de médecins et d'administrateurs de haut niveau, ou simplement 
de héros de séries, on pourrait contribuer à changer les modèles des jeunes : ils sont très réceptifs lorsque la communication est efficace. L'histoire de la fiction cinématographique et des séries américaines en est un exemple, que le cinéma français est encore loin de refléter. Naturellement, les choses ne changeront pas du jour au lendemain, il faut miser sur le long terme.

En ce moment, je travaille avec le scénariste Nicolas Peufaillit, qui a écrit Un prophète (2009) avec Abdel Raouf Dafri, et aussi la série Les Revenants (2012). Nous travaillons sur un projet de série, Autonomia, où on raconte comment la faillite d'un bailleur provoque l'appauvrissement de certaines familles. Tout le monde est un peu pris au piège de cette situation et doit s'organiser pour maintenir le parc de logements. Cela se fonde donc sur la nécessité de se solidariser, de travailler ensemble pour un but commun.

Nous travaillons aussi sur un projet où une femme obèse, qui vit dans un quartier, voudrait « pécho » (sortir avec) son meilleur pote. Le problème est qu'il est Youtubeur et qu'il a un énorme succès auprès des filles sur Internet. Comment peut-elle faire pour le conquérir. Ma démarche consiste dans la mise en scène de problématiques communes de la vie en périphérie, qui ne sont plus liées aux stéréotypes connus, tels ceux du jeune confronté à la police ou à la réussite financière. Avec Nicolas nous voulons multiplier ce type de projets de fictions pour y sensibiliser les jeunes. En 2016, nous organisons, avec le CNC (Centre national du cinéma) et le CGET (Commissariat général à l'égalité des chances et des territoires), cinq rencontres nationales sur le thème «La France de demain. La fiction peut-elle faire évoluer nos quartiers? ». Des professionnels du cinéma iront à la rencontre des publics pour les interroger sur leur vision de la fiction et du cinéma et pour comprendre comment on peut construire des fictions auxquelles les gens pourront s'identifier. Notre défi est de prouver que la fiction peut faire évoluer la société et nos quartiers. On lancera aussi des appels pour des projets de fiction. Et ensuite on accompagnera la réalisation des projets choisis. C'est une première initiative qui je l'espère fera des émules, car je suis convaincu que la fiction peut avoir un impact important sur les publics et peut contribuer à une forme de réappropriation de notre société.

s.c. : Qui sont les lecteurs des «romans de banlieue»? Les habitants des quartiers sensibles les lisent-ils?

R.S. : Quand on écrit sur la banlieue, il ne faut pas espérer avoir du succès en banlieue. Je sais qu'un de mes romans circulera entre une dizaine de personnes tout au plus. Il y a des lecteurs curieux. Mais aujourd'hui, les jeunes vivent dans une culture de la vidéo, de l'instantané: on cherche sur YouTube, on tape un mot-clé et on regarde. Au contraire, prendre un livre et le lire suppose d'y consacrer du temps. Quand j'interviens dans des établissements scolaires je me rends compte que le nombre de pages effraie souvent. Cependant, les jeunes qui entrent dans mes livres disent en apprécier l'oralité et le langage dans lequel ils se reconnaissent. Mais de façon générale ils n'ont plus aucune familiarité avec l'objet livre, qui demande une attention soutenue. Cela correspond aussi à un manque de curiosité, on n'a plus besoin d'être curieux, on a le sentiment d'avoir toute l'information à portée de main. Le livre n'est plus accessible pour le public jeune. Ensuite, au-delà des jeunes, il y a un public qui ne franchit pas le périphérique en termes de littérature : il franchira les frontières en lisant des histoires qui se déroulent au Mexique ou aux États-Unis, parce que c'est une manière de sublimer la réalité, mais lire des histoires qui ont lieu à Saint-Denis, c'est comme regarder les faits divers au journal de 20 heures En gros, je pourrais dire que mes lecteurs se situent 
entre un public qui n'a pas la culture du livre et un public qui considère que je fais de la littérature de "racaille», ce dont je suis en partie responsable, puisque je revendique, en tant qu'écrivain, mon appartenance au $93^{4}$ et que je ne puise pas mes références dans la culture littéraire française.

s.c. : Pour terminer, pouvez-vous nous décrire le projet de la « Dictée des cités »?

R.S.: Depuis août 2013 on organise des dictées géantes dans les banlieues avec l'association "Force des mixités». On utilise des extraits de littérature française classique parce qu'on tient à rendre un hommage à cette littérature qui est la nôtre. Nous avons organisé la première dictée dans un lieu devenu symbolique, à La Dalle d'Argenteuil ${ }^{5}$, avec nos propres moyens. Nous avions quarante chaises et quelques tables. Nous espérions les utiliser toutes, d'autant plus que nous avions invité des médias. Finalement on s'est retrouvé avec 250 personnes qu'il a fallu faire asseoir. J'avais choisi un texte des Trois mousquetaires de Dumas, et cela a été un succès exceptionnel. Un mois plus tard on a reproduit l'expérience, à Saint-Denis, où on a réussi à réunir de nouveau environ 250 personnes. Nous nous sommes rendu compte que quelque chose de nouveau était en train de se dérouler : les gens faisaient la fête, et ont participé même des personnes qui avaient un mauvais rapport avec la dictée. L'un des éléments de la réussite de ce projet est que nous avions décidé d'offrir des lots correspondant aux modèles de consommation partagés par les habitants de la cité, en choisissant de diviser les participants en cinq catégories (primaire, collège, lycée, homme et femme): les gens venaient faire la dictée pour gagner le lot, dans un bon esprit consumériste, mais ils passaient aussi un bon moment et se mettaient à l'épreuve. En mai 2015 on a organisé la plus grande dictée de France, pour la « Dictée des cités " qui a réuni, encore à Saint-Denis, 980 participants. Cette expérience a été impressionnante parce qu'elle a montré que l'on peut dépasser les clivages : autour de la langue française tous les publics se retrouvent. Cela me réconforte sur la possibilité de réunir les gens autour de projets culturels en allant sur le terrain. Même si ce type de projet fortement médiatisé court le risque d'une instrumentalisation politique, assez inévitable, et que pour ma part, je regrette.

\section{NOTES}

1. Brigade anti-criminalité (N.D.L.R.).

2. Ed. Moisson rouge, 2011.

3. Marc Trévidic, ancien juge antiterroriste (N.D.L.R.).

4. Département de la Seine Saint-Denis (N.D.L.R.).

5. Nicolas Sarkozy y avait prononcé la phrase contestée: « Vous en avez assez de cette bande de racailles? On va vous en débarrasser ", le 25 octobre 2005 (N.D.L.R.). 


\section{AUTEUR}

SERENA CELLO

Université de Gênes et Université François-Rabelais de Tours 